\title{
PHOTOIONIZED TOF MASS SPECTROMETRY OF ATOMIC CLUSTERS
}

\author{
Shigeo Maruyama, Hideaki Kinbara, Hideaki Hayashi, \\ and Dai Kimura
}

Departm ent of Mechanical Engineering, The University of Tokyo, Tokyo, Japan

\begin{abstract}
Generation of carbon, silicon, and silver clusters by a laser-vaporization supersonic expansion cluster beam source was studied using a reflectron-type time-of-flight (TOF) mass spectrometer. The atomic cluster was generated by the laser vaporization of a solid sample disk, followed by cooling with supersonic expansion of pulsed helium gas. The neutral cluster, carried by helium gas, was ionized by a fourth harmonic Nd:YAG laser and accelerated by high voltage to the reflectron mass spectrometer. Positive-ion TOF mass spectra of carbon, silicon, and silv er clusters were compared for sev eral cluster source nozzle conditions. Helium gas pressure at the time of $v$ aporization of solid material played the key role in cluster size range obtained. The effect of pressure was thoroughly examined for a relativ ely large carbon cluster, which was regarded as fullerene.
\end{abstract}

Atomic and molecular clusters are being recognized as playing an important role in the thin-film deposition process and phase-change phenomena. Furthermore, small clusters are the most adequate system for the verification of quantum molecular dynamics calculations such as the interference of light and matter, since clusters are small atomic systems with physically reasonable boundary conditions. Experimental treatments of such atomic and molecular clusters are now desired. In order to examine such atomic and molecular clusters, we have implemented a laser-vaporization supersonic-expansion cluster beam source with a reflectron-type time-of-flight (TOF) spectrometer. In this article, the design of the cluster beam source and the reflectron TOF spectrometer are described, along with the carbon, silicon, and silver clusters generated. The generation of relatively large carbon clusters known as fullerene are especially emphasized. The effect of cluster source parameters such as nozzle pressure and vaporization laser wavelength on the generated fullerene mass distribution are discussed.

This work was supported by a Grant-in-Aid from the Ministry of Education for Scientific Research from the Ministry of Education, Science and Culture, Japan.

This article was originally presented at the U.S.-Japan Joint Seminar on Molecular and Microscale Transport Phenomena, Santa Barbara, CA, 7-10 August 1996.

Address correspondence to Shigeo Maruyama, Department of Mechanical Engineering, The University of Tokyo, 7-3-1 Hongo, Bunkyo-ku, Tokyo 113, Japan. E-mail: maruyama@photon.t.utokyo.ac.jp 


\section{EXPERIMENTAL APPARATUS AND METHOD}

The schematics of the cluster source apparatus is shown in Figure 1. It is connected to the TOF mass spectrometer in Figure 2. The key design feature is the same as one of the authors' previous work, done with Smalley's group at Rice University [1]. The whole source system is mounted on a 6-in. six-way cross of a UHV chamber which can be pumped to about $10^{-8}$ torr. The very short helium gas pulse of about $50 \mu \mathrm{s}$ duration is injected by the fast pulsed nozzle (Jordan valve) from the top of the chamber. The sample disk is mounted on the sample holder, which is pushed to the nozzle block and can rotate. The solid sample material is vaporized by the laser beam focused to about $1 \mathrm{~mm}$ diameter. Instead of the pressure inside the nozzle, the pressure variation measured at the target disk position is shown in Figure 3. A miniature diaphragm pressure gauge is connected to the nozzle instead of the target disk. Even though the pressure profile is dull due to the small conductance of the 1-mm hole for the laser beam, a qualitative change of the pressure in the nozzle from the trigger to the pulsed valve is observed.

The delay time of the pulsed laser from the pulsed trigger is controlled by the precise delay generator interfaced with a PC. The second harmonic of a Q-switched $\mathrm{Nd}$ :YAG laser $(532 \mathrm{~nm})$ or an optical parametric oscillation (OPO) tunable laser $(500-867 \mathrm{~nm})$ is used as the vaporization laser. The vapor of the sample expands to the atmosphere of helium, and the clusters are formed in the nozzle. Then clusters are carried and cooled by the helium gas flow, which undergoes the supersonic expansion to the vacuum. The cluster beam is then skimmed by a $\phi 2.0-\mathrm{mm}$ skimmer to the reflectron TOF region (shown in Figure 2) underneath the cluster source. When the packet of the cluster beam arrives between the electrodes, the ionization laser (fourth-harmonic Nd:YAG, $266 \mathrm{~nm}$ ) is shot. The ionized cluster is accelerated by the positive high voltage set at the end electrode. The acceleration is achieved by two stages for the higher mass resolution [2], as described later. Then the positively charged cluster beam enters two pairs of ion deflectors, a vertical pair of plates to cancel the kinetic energy of clusters accelerated by the helium flow, and a horizontal pair of plates to adjust the direction of the beam to the center of the reflectron. Here, we need to adjust voltages to the pair of vertical

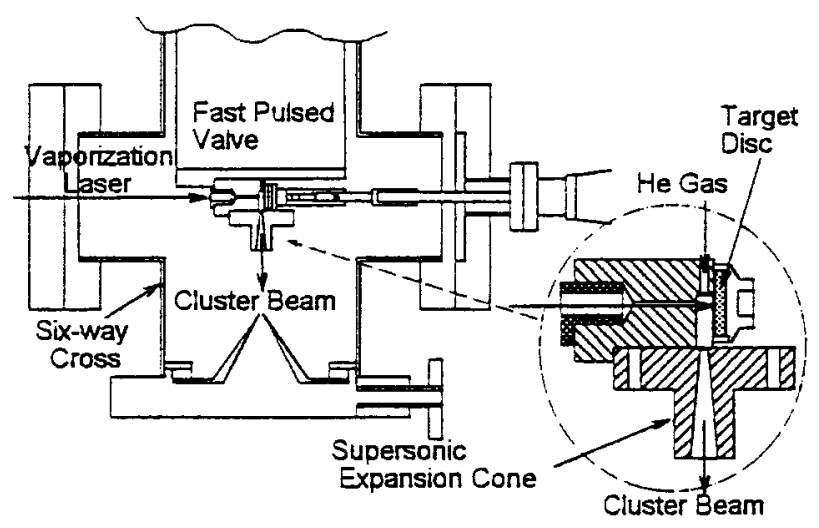

Figure 1. Schematics of cluster beam source apparatus. 


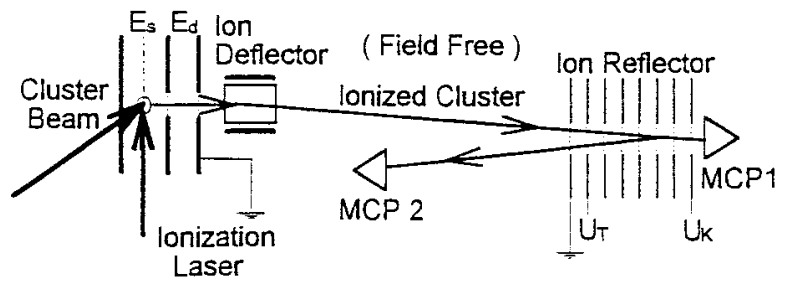

Figure 2. Schematics of reflectron TOF mass spectrometer.

plates according to the mass range. The ion beam is then reflected by the constant electric field (linear gradient of voltage) set at the reflector, which is an array of meshed plates with dividing electric resistance between them. Finally, the ion beam arrives at the ion detector, MCP2 in Figure 2, which is a tandem microchannel plate typically operated at $2-\mathrm{kV}$ bias voltage to obtain a gain of about $10^{8}$. The electric current signal is acquired directly by a digital storage oscilloscope. There is another microchannel plate, MCP1 in Figure 2, behind the reflector in order to constitute the linear TOF spectrometer, with which we can obtain higher signal level but poorer mass resolution.

The timing triggers to the pulsed valve, vaporization-laser Q-switch and ionization-laser Q-switch are controlled by the PC. The total flight time of clusters starting from the ionization is expressed as

$$
\begin{aligned}
& t=\sqrt{\frac{2 m U_{0}}{e}}\left[\frac{1}{E_{s}}\left\{\left(1-k_{1}\right) \sqrt{k-\frac{U_{d}}{U_{0}}}+k_{1} \sqrt{k}\right\}+\frac{L}{2 U_{0} \sqrt{k}}+\right. \\
&\left.\frac{2 d_{T}}{U_{T}}\left(\sqrt{k}-\sqrt{k-\frac{U_{T}}{U_{0}}}\right)+\frac{2 d_{K}}{U_{K}-U_{T}} \sqrt{k-\frac{U_{T}}{U_{0}}}\right]
\end{aligned}
$$

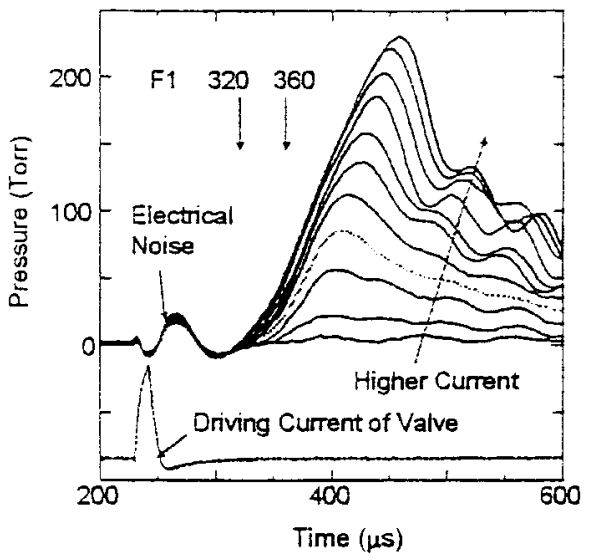

Figure 3. Pressure at the nozzle. 


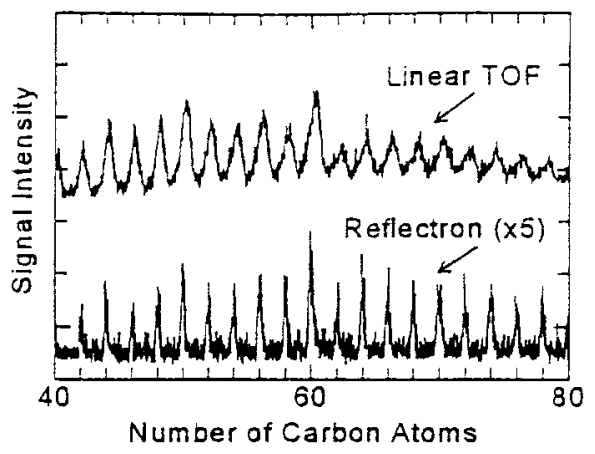

Figure 4. Comparison of linear TOF and reflectron.

where the initial potential of cluster is $U$, mass is $m$, total length of field free region is $L, U=k U_{0}, k_{1}=E_{s} / E_{d}$. The two-stage acceleration and reflectron are designed to minimize the variation of the arrival time $t$ due to the variation of initial potential $U$, which is intrinsic due to the finite volume of ionization region. Thus, $d t / d k=0$ and $d^{2} t / d k^{2}=0$ are the conditions to determine the two-stage voltages and reflectron voltages $[3]$. The mass spectra are typically averaged for 100 times.

The effect of the reflectron is demonstrated in Figure 4. Mass distribution of large carbon clusters including $\mathrm{C}_{60}$ is compared with linear TOF and reflectron TOF. The higher mass resolution by the reflectron is apparent. However, the signal intensity is weaker for the reflectron due to the loss of cluster during the longer and complicated flight pass.

The effect of the ionization fluence is shown in Figure 5. With higher fluence of ionization laser, more fragmentation of cluster is observed. For the carbon clusters, two photons are needed to be ionized. We tried to use the smallest fluence necessary to obtain a signal.

\section{REFLECTRON TOF MASS SPECTRA OF VARIOUS CLUSTERS}

Mass spectra of carbon, silicon, and silver clusters are compared in Figures 6-8. Those cluster distributions were obtained with $532-\mathrm{nm}$ laser vaporization. The carbon cluster distribution obtained from a graphite sample disk is shown in Figure 6. Only even-numbered clusters were observed for positively charged clusters containing more than 30 carbon atoms, and $\mathrm{C}_{60}$ was special as reported previously [4]. The special feature of the carbon cluster is that we could obtain relatively large clusters with less pressure of the nozzle compared with the cases of silicon and silver clusters.

Figure 7 shows a silicon cluster mass spectrum. Clusters up to $\mathrm{Si}_{11}$ are observed in Figure 7. The signal intensity decreased monotonically with cluster size, though $\mathrm{Si}_{6}$ is especially large. This is because of the fragmentation of larger clusters. The effect was demonstrated by experiments with a larger fluence of ionization laser. We observed enhanced $\mathrm{Si}_{1}$ and $\mathrm{Si}_{2}$ signal but decreased intensity for larger clusters. The optimum pressure condition for silicon clusters is much 

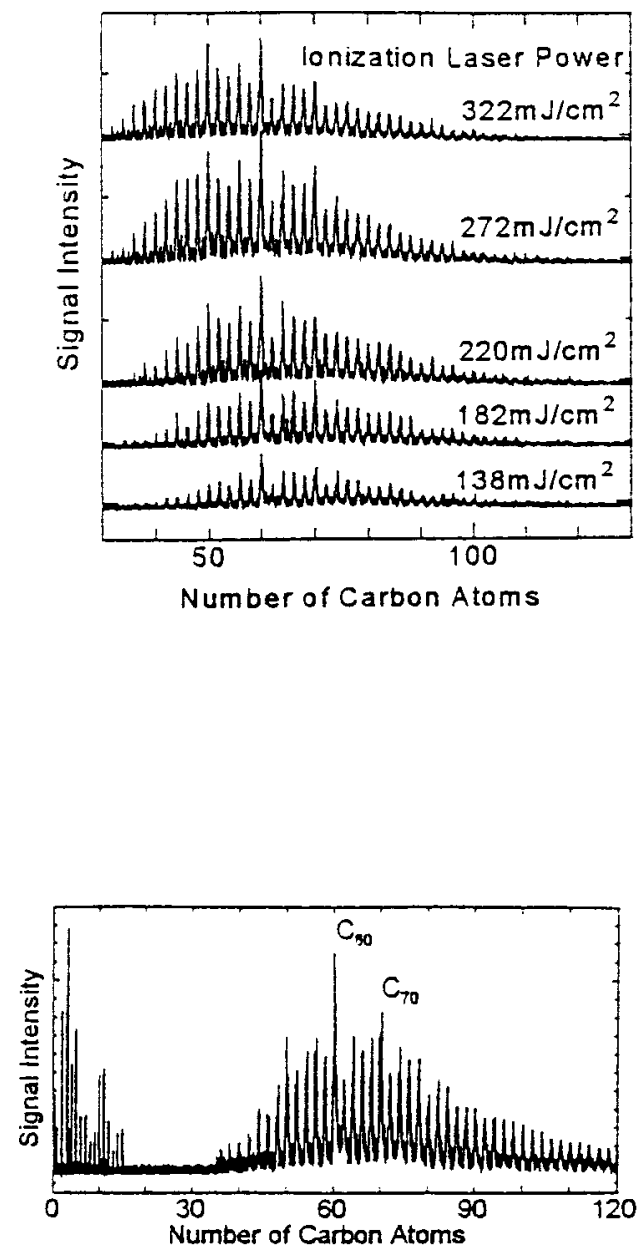

Figure 5. Effect of ionization fluence.

Figure 6. TOF mass spectrum of carbon cluster.

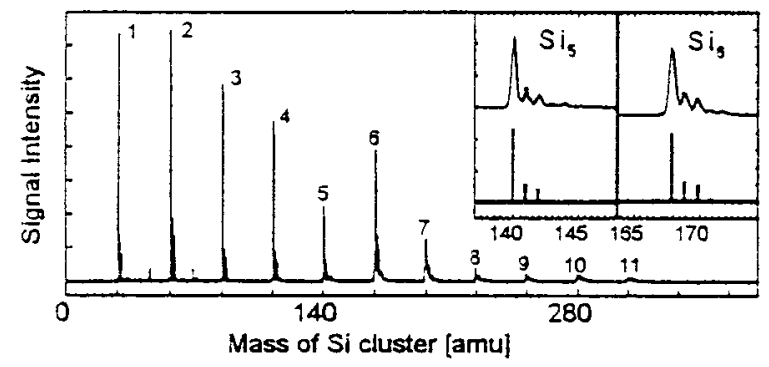

Figure 7. TOF mass spectrum of silicon cluster. 


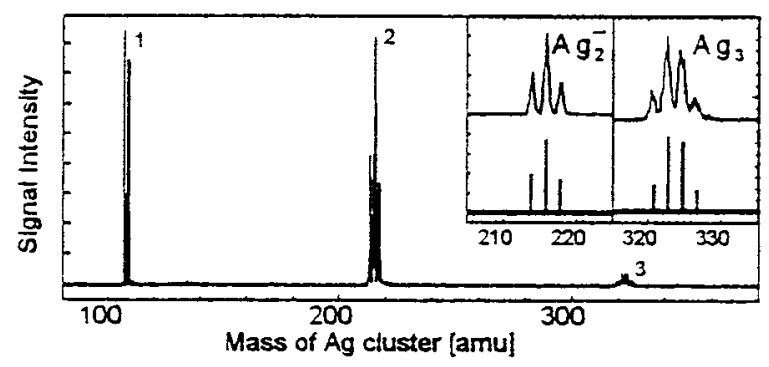

Figure 8. TOF mass spectrum of silver cluster.

higher than for carbon clusters. Each subpeak of the silicon cluster corresponds to the isotope distribution due to the natural abundance of isotopes $\left({ }^{28} \mathrm{Si} 92.23 \%,{ }^{29} \mathrm{Si}\right.$ $\left.4.67 \%,{ }^{30} \mathrm{Si} 3.10 \%\right)$. The comparison of isotope distribution with the theoretical one is demonstrated in the insert for $\mathrm{Si}_{5}$ and $\mathrm{Si}_{6}$.

Figure 8 shows the mass spectrum for small silver clusters. The isotope distribution (natural abundance: ${ }^{107} \mathrm{Ag} 51.84 \%$ and ${ }^{109} \mathrm{Ag} \mathrm{48.16 \%}$ ) is compared in the insert for $\mathrm{Ag}_{2}$ and $\mathrm{Ag}_{3}$. To obtain this silver cluster distribution, higher helium gas pressure and higher power of the ionization laser were necessary compared with silicon clusters.

\section{EFFECT OF CLUSTER SOURCE PARAMETERS ON GENERATION OF FUUERENE}

The effects of cluster source parameters on the generation of fullerene, a carbon cluster in the range $\mathrm{C}_{30}-\mathrm{C}_{130}$, were studied. Figure 9 shows the effect of delay of the Q-switch of vaporization laser. The delay of the Q-switch corresponds to the pressure of the nozzle at the onset of the vaporized plume as shown in Figure 3. The overall signal intensity reaches maximum at about $340 \mu \mathrm{s}$. The pressure in the nozzle at the time of laser shot corresponds to about 150 torr from Figure 3. This optimum pressure of helium is very close to the optimum pressure of the arc-generation method, 100-500 torr. However, the laser ablation fullerene generator works with argon gas at 500 torr. The vaporization laser is $\mathrm{Nd}: \mathrm{YAG}$, second harmonic. The effect of valve current is shown in Figure 10. Higher current corresponds to longer duration of high pressure of the nozzle as shown in Figure 3. The amount of total pressure is indicated in each spectrum as the measure of the valve driving current. Since the onset pressure changes at the same time as the high pressure duration, overall signal intensity is maximum for the optimum current. At the same time, it is apparent that relatively larger clusters become more enhanced for longer duration of high pressure. It seems reasonable to expect larger clusters using the higher pressure.

The effect of the wavelength of the vaporization laser is compared in Figure 11. Keeping the fluence of laser constant, the wavelength was changed in the visible light range, 500-867 $\mathrm{nm}(2.48-1.43 \mathrm{eV})$. Each spectrum is normalized with the height of the $\mathrm{C}_{60}$ signal. No effect of wavelength on the distribution pattern is observed in Figure 11. Even though the vaporization process and clustering process 

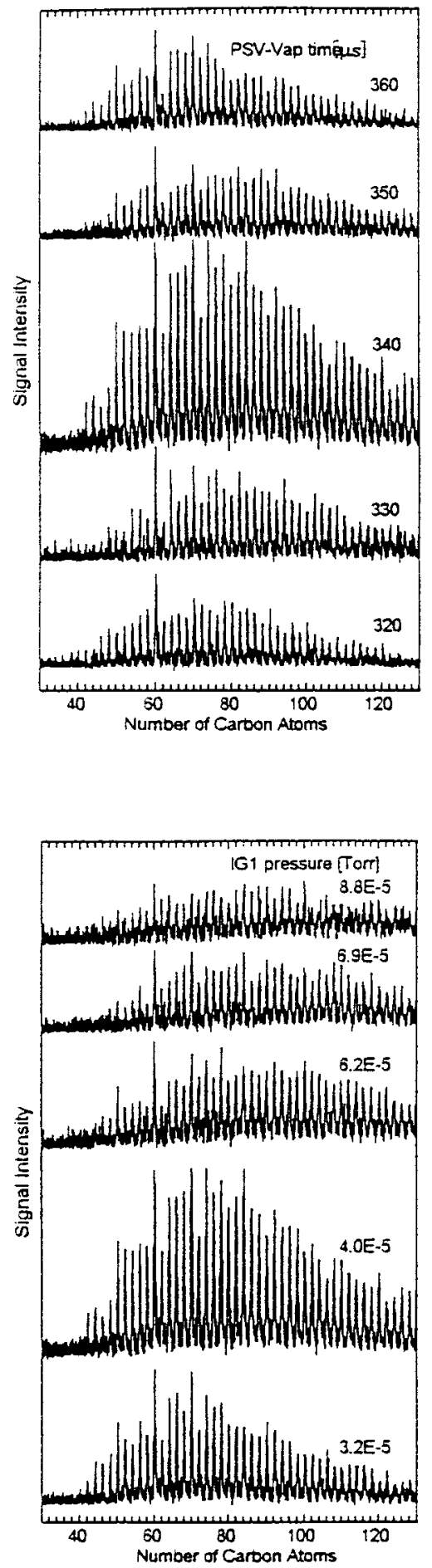

Figure 9. Effect of helium gas pressure.

Figure 10. Effect of helium gas duration. 


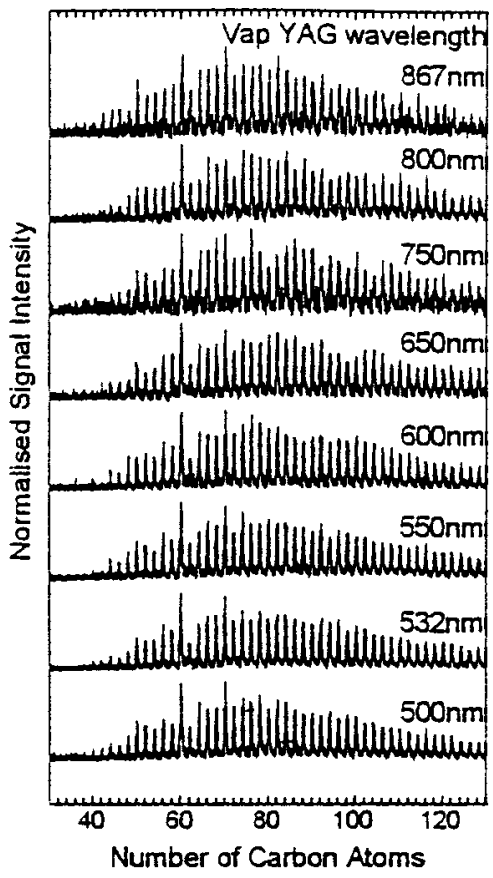

Figure 11. Effect of wavelength of vaporization laser.

is rather complicated because of the formation of plasma, it is suggested that the primary role of the vaporization laser is thermal heating.

\section{REFERENCES}

1. S. Maruyama, L. R. Anderson, and R. E. Smalley, Direct Injection Supersonic Cluster Beam Source for FT-ICR Studies of Clusters, Rev. Sci. Instrum., vol. 61, pp. 3686-3693, 1990.

2. W. C. Wiley and I. H. McLaren, Time-of-Flight Mass Spectrometer with Improved Resolution, Rev. Sci. Instrum., vol. 26, pp. 1150-1157, 1955.

3. H. Shinohara, Laser-Ionization Spectroscopic Studies on Molecular Clusters via Timeof-Flight Mass Spectrometry, Mass Spectrosc., vol. 38, no. 2, pp. 43-63, 1990.

4. H. W. Kroto, J. R. Heath, S. C. O’Brien, R. F. Curl, and R. E. Smalley, C60: Buckminsterfullerene, Nature, vol. 318, pp. 162-163, 1985. 